\title{
Nanoscale
}

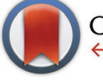

CrossMark

\& click for updates

Cite this: Nanoscale, 2014, 6, 13416

Received 31st July 2014,

Accepted 22nd September 2014

DOI: $10.1039 / c 4 n r 04348 k$

www.rsc.org/nanoscale

\section{Convective polymer assembly for the deposition of nanostructures and polymer thin films on immobilized particles $\uparrow$}

\author{
Joseph J. Richardson, Mattias Björnmalm, Sylvia T. Gunawan, Junling Guo, \\ Kang Liang,\$ Blaise Tardy, Shota Sekiguchi,§ Ka Fung Noi, Jiwei Cui, \\ Hirotaka Ejimaף and Frank Caruso*
}

\begin{abstract}
We report the preparation of polymer particles via convective polymer assembly (CPA). Convection is used to move polymer solutions and cargo through an agarose gel that contains immobilized template particles. This method both coats and washes the particles in a process that is amenable to automation, and does not depend on passive diffusion or electrical currents, thus facilitating incorporation of fragile and nanoscale objects, such as liposomes and gold nanoparticles, into the thin polymer films. Template dissolution leads to the formation of stable polymer particles and capsules.
\end{abstract}

\section{Introduction}

Layer-by-layer (LbL) assembly has been widely used for the engineering of polymer capsules. ${ }^{1-3}$ Recently, we reported a new paradigm for producing $\mathrm{LbL}$ assembled polymer capsules from immobilized template particles. ${ }^{4,5}$ By immobilizing template particles in a porous hydrogel, namely agarose, polymer solutions can be moved through the hydrogel to coat the immobilized particles. The hydrogel can then be dissolved, allowing for recovery of the layered particles, and after template dissolution, robust nano- and micro-capsules can be formed. Thus far, electrophoretic ${ }^{4}$ and immersive ${ }^{5}$ polymer assembly (EPA and IPA, respectively) have been used to coat particles in a rapid or automated fashion, respectively, with various polymer combinations (electrostatically associated synthetic and biopolymers, and hydrogen bonding polymers) and on template particles of different diameters (from approxi-

ARC Centre of Excellence in Convergent Bio-Nano Science and Technology, and the Department of Chemical and Biomolecular Engineering, The University of Melbourne, Parkville, Victoria 3010, Australia. E-mail: fcaruso@unimelb.edu.au $\dagger$ Electronic supplementary information (ESI) available: Detailed experimental/ instrumental information and supporting figures. See DOI: 10.1039/c4nr04348k $\$$ Present address: CSIRO Process Science and Engineering, Clayton, Victoria 3168, Australia

$\S$ Present address: Graduate School of Chemical Sciences and Engineering, Hokkaido University, Sapporo, Japan

ๆ Present address: Institute of Industrial Science, The University of Tokyo, Tokyo, Japan mately $40 \mathrm{~nm}$ to $5 \mu \mathrm{m})$. Both EPA and IPA have unique advantages and approach the comprehensive versatility of conventional LbL assembly, which uses centrifugation and washing to separate unbound polymer from the coated template particles. For example, conventional LbL assembly can use different interactions ${ }^{2,6,7}$ (such as electrostatic, ${ }^{1,8}$ hydrogen bonding, ${ }^{9,10}$ or even host guest ${ }^{11-13}$ ) and different template particles (such as melamine formaldehyde, ${ }^{1}$ polystyrene, ${ }^{1}$ silica, ${ }^{14}$ gold, ${ }^{15}$ and calcium carbonate ${ }^{16}$ of different sizes) for layer build-up. Although choice over templates and polymers provides a broad tool-kit for assembling capsules with different properties, part of the reason LbL capsules have found use in a broad range of fields ranging from biosensing and bioreactions, to drug and vaccine delivery ${ }^{2,6}$ is their capacity to load and release functional units, or "cargo".

For polymer capsules, cargo can be loaded and encapsulated before, during, or after capsule preparation. ${ }^{17}$ Pre-loading can be accomplished by co-precipitating cargo during template formation, or by using templates that can adsorb cargo prior to coating. ${ }^{14,16}$ In contrast, post-loading can be achieved by making the already prepared capsules permeable and resealing the shell after encapsulation. ${ }^{18}$ This can be accomplished by changing the salt concentration, ${ }^{19}$ solvent, ${ }^{20} \mathrm{pH},{ }^{21-24}$ and temperature, ${ }^{25}$ or by cross-linking the capsules. ${ }^{26}$ Pre- and postloading exploit the properties of the template and shell compositions, respectively. Loading during assembly is interesting to explore for next generation film assembly techniques because unique cargo can be loaded, such as sensitive materials that may not be stable during the treatments necessary for pre- or post-loading. For example, liposomes ${ }^{27-29}$ and polymersomes ${ }^{30}$ have been incorporated during layer build-up to allow for the specific encapsulation and protection of biological cargo. Loading sensitive amphiphilic cargo adds an additional benefit, as the hydrophobic compartments of the structures can be further utilized to load hydrophobic molecules. ${ }^{31}$ Therefore, it is important to expand the capacity of layering on immobilized template particles to specifically allow for cargo loading during the assembly process, so that large and/or sensitive cargo can be incorporated into the resultant polymer capsules. 


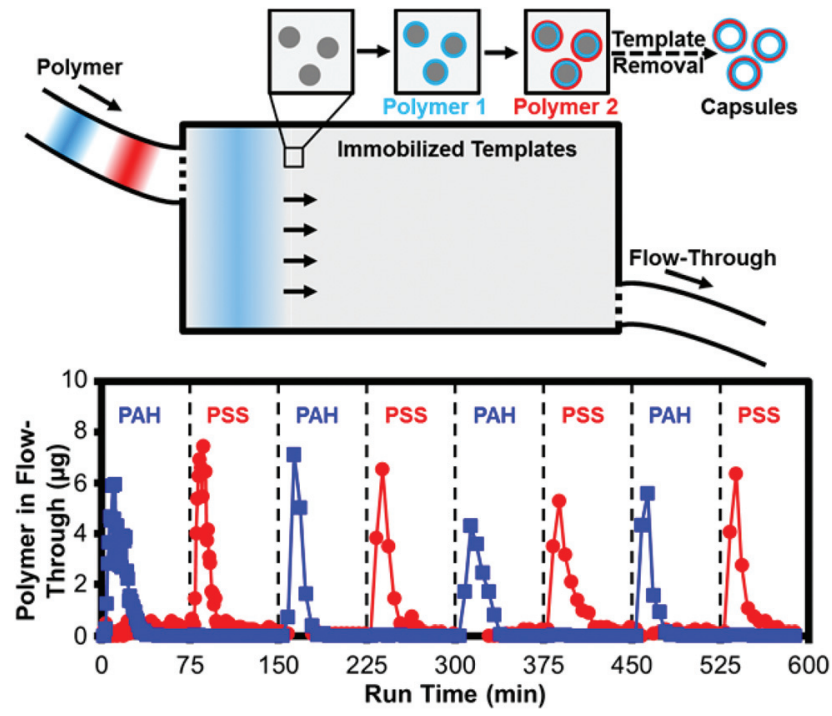

Fig. 1 Schematic illustration of CPA showing polymer flowing into the agarose containing immobilized particles and layer build-up. The resultant LbL core-shell particles can be recovered from the agarose, and the templates can be removed to yield LbL capsules. The polymer flowthrough was collected and quantified over four PAH/PSS cycles. Magnified portions of the first bilayer can be seen in Fig. S2. $\dagger$

Herein, we deposit polymers and cargo on immobilized particles by using convective polymer assembly (CPA), or polymer assembly using a directional flow (Fig. 1). CPA allows for a highperformance liquid chromatography (HPLC) like set-up, using syringe pumps and standard tubing (Fig. S1†), to move the polymer, cargo, and washing solutions past the immobilized template particles, thereby depositing the polymer or cargo on the templates and subsequently removing the unbound polymer. CPA was chosen as the random diffusion associated with IPA would prevent large cargo $^{32}$ from diffusing through the agarose to achieve deposition on immobilized templates within a reasonable time scale. Similarly, the electric current used in EPA could destabilize sensitive cargo such as liposomes. ${ }^{33,34}$ Additionally, the template choice for CPA was expanded by using calcined calcium carbonate $\left(\mathrm{CaCO}_{3}\right)$ particles to form replica particles. Hence, CPA complements IPA and EPA by: (i) allowing deposition of larger cargo by not relying on diffusion; (ii) allowing deposition of sensitive cargo, as no electric current is needed; and (iii) allowing the use of a wider range of templates, as convection does not limit the types of buffers that can be used in the same way as an electrical system, such as EPA, does. CPA therefore addresses some of the challenges associated with IPA and EPA, while retaining advantages such as easeof-use and high amenability to automation.

\section{Experimental details}

\section{Preparation of agarose containing particle templates}

$1 \%, 2 \%$, and $4 \%$ ultra-low gelling agarose gels were prepared in ultrapure water. $150 \mu \mathrm{L}$ of $50 \mathrm{mg} \mathrm{mL}^{-1}$ silica particles $(1.11$ or $4.99 \mu \mathrm{m}$ in diameter) or $150 \mu \mathrm{L}$ of $10 \mathrm{mg} \mathrm{mL}^{-1}$ calcined
$\mathrm{CaCO}_{3}$ particles were added for each $1.5 \mathrm{~mL}$ of liquid gel solution. This liquid agarose particle solution was then pipetted into 3DKUBEs (Kiyatec, Greenville, SC, USA).

\section{Convective polymer assembly (CPA)}

The 3DKUBE was attached to a 6-way valve and syringe pump (Fig. S1 $\dagger$ ). The syringe was loaded with ultrapure water and the pump flow rate was set to $100 \mu \mathrm{L} \mathrm{min}^{-1}$. Poly(allylamine hydrochloride) (PAH, $\sim 15 \mathrm{kDa}$ ) or poly(styrene sulfonate) (PSS, $\sim 15 \mathrm{kDa})$, in $0.5 \mathrm{M}$ sodium chloride $(\mathrm{NaCl})$ at $\mathrm{pH} 7$, with a polymer concentration of $0.1 \mathrm{mg} \mathrm{mL}^{-1}$, was loaded into the loading tube and the 6-way valve was switched from "load" to "inject." $2 \mathrm{~mL}$ of polymer solution was loaded through the loading tube to ensure that the polymer remained at the required concentration and was not diluted. The process was repeated for each polymer layer until the desired number of layers was achieved. Polymer was injected every $75 \mathrm{~min}$ for the initial characterization of the flow-through, and every $45 \mathrm{~min}$ for all subsequent layering experiments.

\section{Cargo loading}

The gold nanoparticles (Au NPs; $15 \mathrm{~nm}$ in diameter) or liposomes ( 100 nm in diameter) were loaded similarly to PAH and PSS, except for the buffers used. The Au NPs were dispersed in ultrapure water and the liposomes were dispersed in HEPES buffer (10 mM HEPES, $150 \mathrm{mM} \mathrm{NaCl}, \mathrm{pH}$ 7.4). The Au NPs were loaded after 3.5 bilayers of PAH/PSS and the liposomes were loaded after 4 bilayers of PAH/PSS, and both were finally capped with one bilayer of PAH/PSS.

\section{Sample recovery}

The agarose containing layered particles was removed from the 3DKUBE and placed into a microcentrifuge tube. Ultrapure water was added to the tube, which was heated $\left(45^{\circ} \mathrm{C}\right)$ until the agarose dissolved. The melted solution was centrifuged at $37^{\circ} \mathrm{C}$ at $800 \mathrm{~g}$ for $30 \mathrm{~s}$ to pellet the particles. The supernatant was removed and discarded. The particles were washed six times to remove the excess agarose and finally resuspended in ultrapure water.

\section{Core removal}

$300 \mu \mathrm{L}$ of hydrofluoric acid (5 M HF) or $40 \mathrm{mM}$ sodium acetate, $\mathrm{pH} 4$ was added to the layered particles, and incubated for $5 \mathrm{~min}$ to remove the silica or $\mathrm{CaCO}_{3}$, respectively. [Caution! $H F$ is highly toxic. Extreme care should be taken when handling HF solution.] The capsules or replica particles were pelleted at $2700 \mathrm{~g}$ for $3 \mathrm{~min}$, the supernatant was removed and they were then washed with ultrapure water three times to ensure that the HF or sodium acetate was fully removed.

Additional experimental details can be found in the ESI. $\dagger$

\section{Results and discussion}

Firstly, we investigated the behavior of the agarose containing silica particles, and the movement of polymers through the 
agarose under convection. Note that columns could also be used with the primary driving forces being gravity and the capillary effect (data not shown), but our analysis hereafter is focused on the HPLC-like set-up (Fig. S1 $\dagger$ ). We observed that

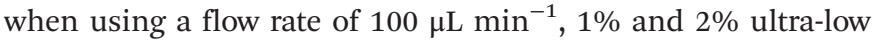
gelling agarose gels would liquefy and flow out of the 3DKUBE during CPA, and we therefore restricted our analysis to $4 \%$ gels. The modular set-up and constant flow rate let us investigate the movement of polymer into and out of the agarose by analyzing fractions of the flow-through (Fig. 1 and S2 $\dagger$ ). The UV-Vis absorbance of the flow-through was used to quantify the amount of polymer adsorbed onto the immobilized particles. An average of $\sim 10 \mu \mathrm{g}$ of PAH and $\sim 10 \mu \mathrm{g}$ of PSS was deposited for each bilayer. Similar values from literature (i.e., 8-16 $\mu \mathrm{g}$ for PAH and 9-12 $\mu \mathrm{g}$ for PSS) are attained when values are normalized to the approximate surface area of the particles $\left(\sim 19 \mathrm{~cm}^{2}\right){ }^{1,35,36}$ This suggests that CPA provides a similar coating coverage to conventional (i.e., non-flow adsorption/ centrifugation/wash) LbL assembly. The polymer reached its peak in the flow-through roughly $10 \mathrm{~min}$ after injection, which corresponds to $1 \mathrm{~mL}$ of polymer solution. This is the same volume as the agarose, suggesting that the polymer solution was flowing through the agarose matrix. The polymer solution reached background levels, corresponding to less than $0.1 \mu \mathrm{g}$ of PAH and less than $0.4 \mu \mathrm{g}$ of PSS, 30-45 min after injection. This equates to 3 to $4 \mathrm{~mL}$ of washing volume, which is comparable to the washing procedure for conventional centrifugation/ wash LbL assembly (3 to 4 wash cycles). Characterization of the flow-through demonstrated that the process could be sped up by injecting the polymer solutions $45 \mathrm{~min}$ after one another, and indicates that even shorter times should be possible with higher flow rates. This injection time (45 min) was used for all subsequent experiments.

By removing and sectioning the agarose prior to washing, it was observed that the concentration of polymer reduced around the template particles from as far away as $\sim 2 \mu \mathrm{m}$ (Fig. S3 $\dagger$ ). Polymer build-up on the immobilized template particles during CPA was characterized using fluorescently labeled PAH. Flow cytometry showed a linear fluorescence growth for polymer build-up, and after four bilayers, capsules were obtained (Fig. 2a). Height analysis using atomic force microscopy (AFM) revealed a bilayer thickness of $\sim 2.5 \mathrm{~nm}$ (Fig. $2 \mathrm{~b}$ ), which corresponds well with the literature value for four bilayer PAH/PSS capsules. ${ }^{37}$ Interestingly, this is an intermediary value compared to films formed with EPA and IPA, which both also use immobilized template particles. EPA yields roughly twice the thickness $(\sim 5 \mathrm{~nm})$ for each bilayer, ${ }^{4}$ while IPA gives half $(\sim 1.25 \mathrm{~nm})$ for each bilayer. ${ }^{5}$ Together with the flow-through analysis, the height measurements demonstrate that CPA is comparable to conventional polymer assembly techniques. Energy-dispersive X-ray (EDX) mapping was also used to confirm the presence of elements unique to each polymer (Fig. $2 \mathrm{c}$ and d). The capsules contained nitrogen from PAH and sulfur from PSS, demonstrating that both polymers were present.

The constant flow of water associated with CPA was then used for the deposition of larger objects into the polymer
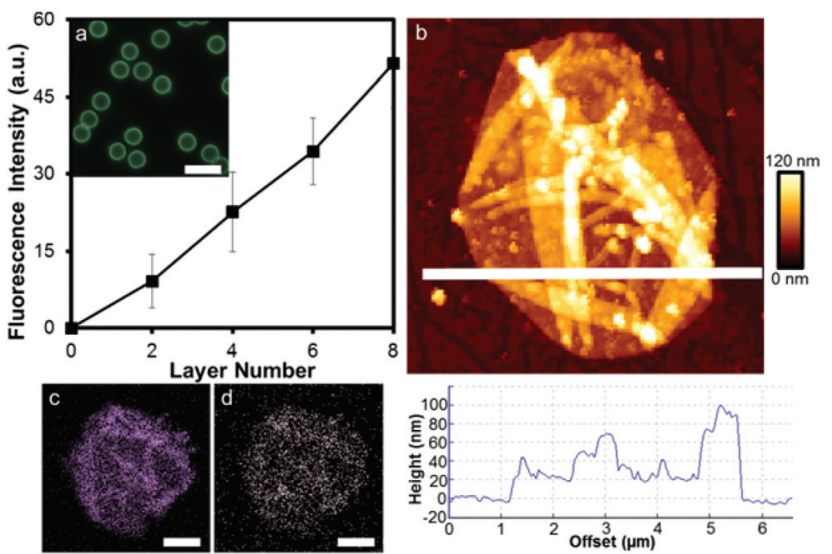

Fig. 2 Layer build-up and characterization of four bilayer PAH/PSS capsules. (a) Fluorescence intensity was measured for core-shell particles after the deposition of each bilayer. (Inset) Fluorescently labeled capsules prepared via CPA using PAH-FITC. (b) AFM image and height profile of a capsule. EDX mapping of a capsule for (c) sulfur and (d) nitrogen. The scale bars are (a) $10 \mu \mathrm{m}$, and (c) and (d) $1 \mu \mathrm{m}$.

films. This is possible due to the relatively large pore-size associated with agarose $(\sim 50 \mathrm{~nm} \text { to } 10 \mu \mathrm{m})^{5}$ and that the flow in CPA is unidirectional. Au NPs (Fig. 3a) were loaded as a proof of concept. Au NPs have previously been incorporated in capsules to allow for photo-induced therapeutic release. ${ }^{38}$ The Au NP-loaded capsules were robust and stable in water, as observed by fluorescence microscopy (Fig. 3b). The Au NPs were detected with EDX (Fig. S4 $\dagger$ ) and more accurately, EDX mapping was used to confirm that the gold was present in the capsules (Fig. 3c). By weight, the gold to sulfur ratio was $1: 399$,

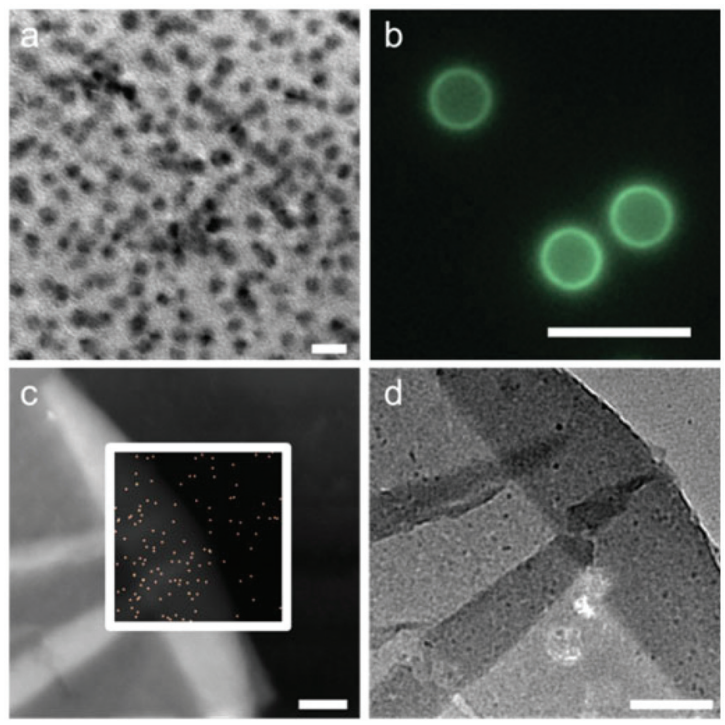

Fig. 3 PAH/PSS capsules loaded with Au NPs via CPA. (a) TEM image of Au NPs. (b) Fluorescence microscopy image of the Au NP-loaded capsules. PAH-FITC was used as the fluorescent polymer. (c) High angle annular dark-field (HAADF) image of a capsule, inset with EDX mapping for gold. (d) TEM image of the same capsule in (c) with Au NPs visible. The scale bars are (a) $20 \mathrm{~nm}$, (b) $10 \mu \mathrm{m}$, and (c) and (d) $200 \mathrm{~nm}$. 
indicating that $\sim 25 \mathrm{ng}$ of Au NPs was deposited, corresponding to approximately $70 \mathrm{Au}$ NPs per capsule. The Au NPs present in the capsule wall were also imaged with TEM (Fig. 3d). The loading of Au NPs demonstrated the capacity of CPA to move and load solid structures larger than standard polymers.

Next, liposomes were loaded into the capsule shell to test the loading of more fragile cargo. Loading was detected through fluorescence microscopy and indirectly through the loading of hydrophobic cargo into the liposomes. The liposomes were labelled with a fluorescent phospholipid during formation, ${ }^{27}$ and consequently the fluorescence was visible in the capsule shell (Fig. 4).

Additionally, a hydrophobic dye (naphthofluorescein) was loaded into the liposomes of both core-shell particles (Fig. 5) and capsules (Fig. S5 $\dagger$ ), confirming the presence of the liposomes. The fluorescence of the dye became concentrated around the core-shell particles due to the dye migrating into the liposomes, which also greatly reduced the fluorescence intensity of the solution. Similar results were seen for the capsules (Fig. S5 and S6†). For both core-shell particles and capsules, the addition of Triton-X-100 disrupted the liposomal structures and allowed the dye to escape back into the solution. The loading of both the Au NPs and the liposomes demonstrated that larger cargo can be successfully incorporated into the capsules prepared from immobilized template particles using CPA.
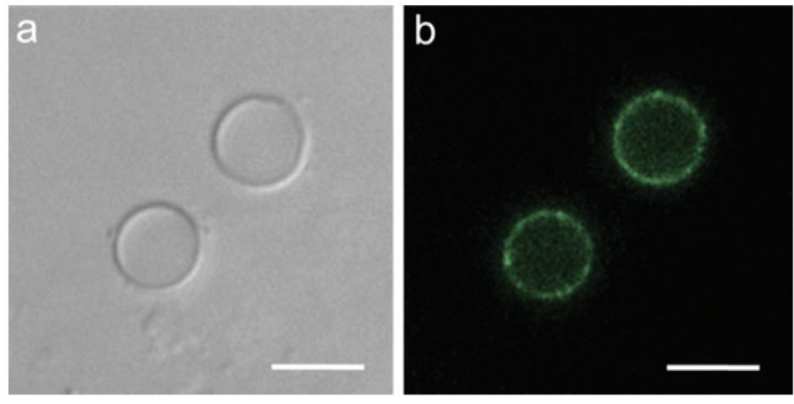

Fig. 4 PAH/PSS capsules loaded with fluorescently labelled liposomes via CPA. Images of capsules acquired using (a) differential interference contrast (DIC) microscopy, and (b) fluorescence microscopy. The scale bars are $5 \mu \mathrm{m}$.
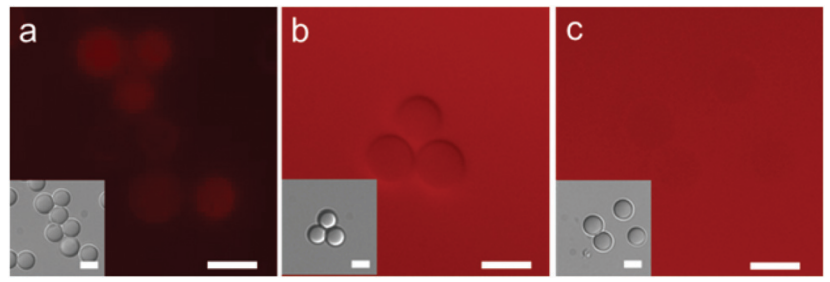

Fig. 5 Loading of PAH/PSS-liposome core-shell particles with a hydrophobic dye, naphthofluorescein. Fluorescence microscopy images of $\mathrm{PAH} / \mathrm{PSS}$-liposome core-shell particles with the corresponding DIC images in the insets: (a) before and (b) after Triton X-100 treatment. (c) Control, liposome-free PAH/PSS core-shell particles after the addition of naphthofluorescein. The scale bars are $5 \mu \mathrm{m}$.
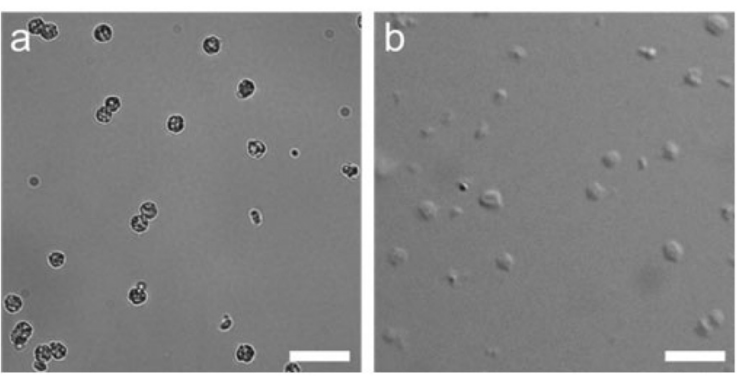

Fig. 6 Polymer replica particles prepared from calcined $\mathrm{CaCO}_{3}$ template particles via CPA. (a) DIC image of the calcined $\mathrm{CaCO}_{3}$ template particles. (b) PAH/PSS replica particles after template removal, produced via CPA. The scale bars are $10 \mu \mathrm{m}$.

Finally, to demonstrate the versatility of CPA being applicable to other template particles, mesoporous calcined $\mathrm{CaCO}_{3}$ particles were used (Fig. 6a and $\mathrm{S}_{7}^{+}$). $\mathrm{CaCO}_{3}$ particles are of particular interest for CPA as the acetate buffers used in EPA readily degrades $\mathrm{CaCO}_{3}{ }^{4}$ Using CPA, 4 bilayers of PAH/PSS were deposited on the $\mathrm{CaCO}_{3}$ particles, after which the $\mathrm{CaCO}_{3}$ core was removed using acetate buffers. The resulting polymer replica particles resembled particles more so than capsules (Fig. 6b). The use of $\mathrm{CaCO}_{3}$ particles as templates greatly extends the application of CPA because the formation of $\mathrm{CaCO}_{3}$ particles can be performed in the presence of therapeutics, which would allow CPA capsules and particles to be pre-loaded.

\section{Conclusions}

This study highlights the use of an immobilizing matrix as an appropriate paradigm from which polymer assembly and cargo loading can be performed for the preparation of engineered particles. We have demonstrated the use of a directional flow for depositing polymers, nanoparticles, and liposomes onto immobilized template particles for the formation of microcapsules and polymer replica particles. Convection allows for the layering of larger materials (such as Au NPs and liposomes) that would not passively diffuse through the agarose matrix on a reasonable timescale. The shell thickness of the capsules prepared via CPA falls in between the thicknesses for capsules prepared via EPA and IPA, and is similar to those prepared by conventional (i.e., adsorption/centrifugation/wash) LbL assembly. ${ }^{37}$ Additionally, porous template particles, namely $\mathrm{CaCO}_{3}$, were used for the formation of polymer replica particles. This technique has the potential to be incorporated into macro- and microfluidic systems, ${ }^{39}$ thereby greatly expanding the variety of equipment and approaches applicable towards the coating of particulate templates. This includes the use of commercially available computer-controlled automatic systems for loading and injection, commonly used in HPLC-setups, which could allow an arbitrary number of layers to be programmed and then performed through repeated automatic loading/injection cycles requiring little to no hands-on time. This would also allow for automation and high-throughput 
studies to be conducted in parallel. Similarly, higher flow rates and reaction chambers of different dimensions could be used to speed up the layering and washing times. Furthermore, CPA could be combined with $\mathrm{EPA}^{4}$ and $\mathrm{IPA}^{5}$ to allow for the benefits of each technique to be used when necessary. For example, thick polymer layers could be deposited using EPA, CPA could be used to add cargo, and finally IPA could be used to autonomously coat different batches at the same time.

\section{Acknowledgements}

This work was supported by the Australian Research Council (ARC) under the Australian Laureate Fellowship (F.C., FL120100030) and the Super Science Fellowship (F.C., FS110200025) schemes as well as the Australian Government through an Australian Postgraduate Award (M.B.) and an International Postgraduate Research Scholarship (M.B.). This research was also conducted and funded by the ARC Centre of Excellence in Convergent Bio-Nano Science and Technology (project number CE140100036).

\section{Notes and references}

1 F. Caruso, R. A. Caruso and H. Möhwald, Science, 1998, 282, 1111-1114.

2 S. De Koker, R. Hoogenboom and B. G. De Geest, Chem. Soc. Rev., 2012, 41, 2867-2884.

3 Y. Yan, M. Björnmalm and F. Caruso, Chem. Mater., 2014, 26, 452-460.

4 J. J. Richardson, H. Ejima, S. L. Lörcher, K. Liang, P. Senn, J. Cui and F. Caruso, Angew. Chem., Int. Ed., 2013, 52, 64556458.

5 J. J. Richardson, K. Liang, K. Kempe, H. Ejima, J. Cui and F. Caruso, Adv. Mater., 2013, 25, 6874-6878.

6 C. S. Peyratout and L. Dahne, Angew. Chem., Int. Ed., 2004, 43, 3762-3783.

7 K. Ariga, J. P. Hill and Q. Ji, Phys. Chem. Chem. Phys., 2007, 9, 2319-2349.

8 E. Donath, G. B. Sukhorukov, F. Caruso, S. A. Davis and H. Möhwald, Angew. Chem., Int. Ed., 1998, 37, 2201-2205.

9 Y. Zhang, S. Yang, Y. Guan, W. Cao and J. Xu, Macromolecules, 2003, 36, 4238-4240.

10 V. Kozlovskaya, S. Ok, A. Sousa, M. Libera and S. A. Sukhishvili, Macromolecules, 2003, 36, 8590-8592.

11 Z. Wang, Z. Feng and C. Gao, Chem. Mater., 2008, 20, 41944199.

12 G. V. Dubacheva, P. Dumy, R. Auzély, P. Schaaf, F. Boulmedais, L. Jierry, L. Coche-Guerente and P. Labbé, Soft Matter, 2010, 6, 3747-3750.

13 O. Crespo-Biel, B. Dordi, D. N. Reinhoudt and J. Huskens, J. Am. Chem. Soc., 2005, 127, 7594-7600.

14 Y. Wang, A. Yu and F. Caruso, Angew. Chem., Int. Ed., 2005, 117, 2948-2952.
15 D. C. Zhiyong Poon, X. Zhao and P. T. Hammond, ACS Nano, 2011, 5, 4284-4292.

16 G. B. Sukhorukov, D. V. Volodkin, A. M. Günther, A. I. Petrov, D. B. Shenoy and H. Möhwald, J. Mater. Chem., 2004, 14, 2073-2081.

17 B. G. De Geest, N. N. Sanders, G. B. Sukhorukov, J. Demeester and S. C. De Smedt, Chem. Soc. Rev., 2007, 36, 636-649.

18 E. Kharlampieva, V. Kozlovskaya and S. A. Sukhishvili, Adv. Mater., 2009, 21, 3053-3065.

19 A. A. Antipov, G. B. Sukhorukov, S. Leporatti, I. L. Radtchenko, E. Donath and H. Möhwald, Colloids Surf., A, 2002, 198, 535-541.

20 Y. Lvov, A. A. Antipov, A. Mamedov, H. Möhwald and G. B. Sukhorukov, Nano Lett., 2001, 1, 125-128.

21 G. B. Sukhorukov, A. A. Antipov, A. Voigt, E. Donath and H. Möhwald, Macromol. Rapid Commun., 2001, 22, 44-46.

22 S. Y. Yang, D. Lee, R. E. Cohen and M. F. Rubner, Langmuir, 2004, 20, 5978-5981.

23 E. Kharlampieva and S. A. Sukhishvili, Langmuir, 2004, 20, 9677-9685.

24 N. G. Balabushevich, O. P. Tiourina, D. V. Volodkin, N. I. Larionova and G. B. Sukhorukov, Biomacromolecules, 2003, 4, 1191-1197.

25 J. F. Quinn and F. Caruso, Langmuir, 2004, 20, 20-22.

26 H. Zhu and M. J. McShane, Langmuir, 2005, 21, 424-430.

27 B. Städler, A. D. Price, R. Chandrawati, L. Hosta-Rigau, A. N. Zelikin and F. Caruso, Nanoscale, 2009, 1, 68-73.

28 B. Städler, R. Chandrawati, A. D. Price, S.-F. Chong, K. Breheney, A. Postma, L. A. Connal, A. N. Zelikin and F. Caruso, Angew. Chem., Int. Ed., 2009, 48, 4359-4362.

29 R. Chandrawati, P. D. Odermatt, S.-F. Chong, A. D. Price, B. Städler and F. Caruso, Nano Lett., 2011, 11, 4958-4963.

30 H. Lomas, A. P. R. Johnston, G. K. Such, Z. Zhu, K. Liang, M. P. van Koeverden, S. Alongkornchotikul and F. Caruso, Small, 2011, 7, 2109-2119.

31 L. Hosta-Rigau, B. Städler, Y. Yan, E. C. Nice, J. K. Heath, F. Albericio and F. Caruso, Adv. Funct. Mater., 2010, 20, 59-66.

32 A. Pluen, P. A. Netti, R. K. Jain and D. A. Berk, Biophys. J., 1999, 77, 542-552.

33 D. Dimitrov and M. Angelova, J. Electroanal. Chem., 1988, 253, 323-336.

34 P. S. O'Shea, G. Petrone, R. Casey and A. Azzi, Biochem. J., 1984, 219, 719-726.

35 Z. Feldötö, I. Varga and E. Blomberg, Langmuir, 2010, 26, 17048-17057.

36 Y. Lvov, K. Ariga, M. Onda, I. Ichinose and T. Kunitake, Colloids Surf., A, 1999, 146, 337-346.

37 S. Leporatti, A. Voigt, R. Mitlohner, G. Sukhorukov, E. Donath and H. Möhwald, Langmuir, 2000, 16, 40594063.

38 A. S. Angelatos, B. Radt and F. Caruso, J. Phys. Chem. B, 2005, 109, 3071-3076.

39 M. Björnmalm, Y. Yan and F. Caruso, J. Controlled Release, 2014, 190, 139-149. 